\title{
Ecología trófica de la lechuza del campanario (Tyto furcata) en las Yungas del noroeste argentino
}

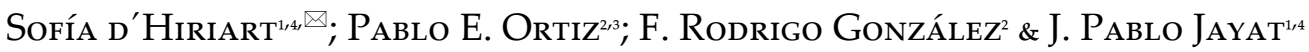 \\ ${ }^{1}$ Instituto de Ambiente de Montaña y Regiones Áridas (IAMRA), Universidad Nacional de Chilecito (UNdeC), Chilecito, La \\ Rioja, Argentina. ${ }^{2}$ Facultad de Ciencias Naturales e Instituto Miguel Lillo, Universidad Nacional de Tucumán, San Miguel \\ de Tucumán, Tucumán, Argentina. ${ }^{3}$ Instituto Superior de Correlación Geológica (INSUGEO, CONICET), San Miguel de \\ Tucumán, Tucumán, Argentina. ${ }^{4}$ Instituto de Ecología Regional, Universidad Nacional de Tucumán y CONICET, Yerba \\ Buena, Argentina.
}

\begin{abstract}
Resumen. Existen pocos antecedentes sobre los hábitos tróficos de Tyto furcata en el noroeste argentino, y ninguno en la ecorregión de las Yungas. A partir del análisis de egagrópilas, caracterizamos la dieta de esta rapaz en seis localidades de las Yungas de Argentina. Documentamos la composición y la diversidad de los ítems presa y analizamos los parámetros tróficos en el contexto de los diferentes grados de transformación de la vegetación. El estudio incluyó una caracterización general y un análisis estacional. Los roedores constituyeron un $98 \%$ del total de presas y fueron los que más aportaron a la dieta en términos de biomasa. Los valores de amplitud de nicho trófico (ANT) y del nicho trófico estandarizado fueron más elevados en comparación con los observados en otras áreas de Argentina. Observamos cambios estacionales importantes en la composición de especies para cada localidad. La ANT fue mayor durante la estación húmeda y el solapamiento trófico entre estaciones fue relativamente alto. No observamos una relación clara entre el grado de modificación antrópica y los parámetros tróficos. Nuestros resultados coinciden con los reportados para América del Sur, con un espectro relativamente amplio de presas y una fuerte selección por roedores cricétidos. Algunas localidades mostraron una tendencia a la disminución de ANT durante la estación seca, lo que reflejaría las variaciones estacionales en la abundancia de pequeños mamíferos, que muestran mayores tamaños poblacionales en invierno. Esto obligaría a la lechuza a adoptar una dieta más generalista en el verano. En estas localidades, la proporción de los diferentes usos del suelo no parece tener una incidencia importante sobre los parámetros tróficos analizados, al menos en nuestra escala de análisis. Aunque nuestros resultados constituyen un punto de partida importante para el estudio del nicho trófico de Ty to furcata en Yungas, resulta evidente la necesidad de estudios adicionales para dilucidar numerosos aspectos vinculados a la ecología de esta rapaz.
\end{abstract}

[Palabras clave: ecorregión de las Yungas, Strigiformes, dieta, pequeños mamíferos, amplitud de nicho trófico]

\begin{abstract}
Trophic ecology of the barn owl (Tyto furcata) in the Yungas of northwestern Argentina. There is few information on the trophic habits of Tyto furcata in northwestern Argentina, and none in Yungas ecoregion. From pellets analyses, we characterized the diet of this raptor in six localities from Yungas of Argentina. We documented the diversity and composition of the prey-items and analyzed trophic parameters in the context of different degrees of natural cover transformation. The study includes a general characterization and a seasonal analysis. Rodents constituted $98 \%$ of the total preys and were the prey items which contributed the most to the diet in terms of biomass. The values for food niche breadth (FNB) and the standardized food niche breadth were higher in comparison with those observed in other areas of Argentina. We observed key seasonal changes in species composition in each locality. FNB was higher during the wet season and the trophic overlap among seasons was relatively high. The relationship between the degree of anthropic modification and trophic parameters was weak. Our results agree with those reported for South America, with a relatively broad spectrum of prey and a marked selectivity for cricetid rodents. Some localities exhibited a trend towards the reduction of FNB during the dry season, which might reflect the seasonal variation in the abundance of small mammals, with higher population sizes in the dry season. This would force the barn owl to adopt a more generalist diet in summer. In the studied localities, the proportion of land use does not seem to be relevant on the trophic parameters assessed, at least at this scale of analysis. Although our results constitute an important starting point for the study of the food niche of Tyto furcata in the Yungas, is evident the need for additional studies to elucidate many aspects related to the ecology of this raptor.
\end{abstract}

[Keywords: Yungas ecoregion, Strigiformes, diet, small mammals, food-niche breadth]

Editor asociado: David Bilenca

\footnotetext{
sofiadiriart@gmail.com
} 


\section{INTRODUCCIÓN}

Los hábitos alimentarios de Tyto furcata han sido documentados ampliamente en diversas regiones de América (e.g., Jaksic et al. 1981; Massoia 1987, 1988; David 1996; Massoia et al. 1997a,b, 1999; Bellocq 2000; Pardiñas and Cirignoli 2002; Álvarez Castañeda et al. 2004). Esta rapaz fue caracterizada en América del Sur como un depredador especialista/oportunista en pequeños mamíferos (principalmente roedores y pequeños marsupiales), con otros ítems alternativos incorporados en proporciones menores, tales como aves, pequeños reptiles, anfibios e insectos (Bellocq 1998; Bó et al. 2007).

La mayor parte de las contribuciones que documentan la dieta de Tyto furcata en Argentina fueron realizadas en agroecosistemas (González Fischer et al. 2012 y referencias allí contenidas) y/o en ambientes naturales abiertos de la región pampeana, y son escasos los estudios de este tipo llevados a cabo en áreas boscosas subtropicales (Bellocq 2000; Pardiñas and Cirignoli 2002; Bó et al. 2007). Para la región noroeste de este país existen pocos antecedentes sobre este tópico (e.g., Oviedo de la Vega 1962; Soncini et al. 1985; Massoia 1988; Gómez et al. 2012; Nanni et al. 2012), ninguno de ellos centrado en poblaciones de Tyto furcata de ambientes boscosos de la ecorregión de las Yungas. En la actualidad, este ambiente está amenazado por distintos factores, en particular por la transformación del bosque a otros usos del suelo, lo que incluye el aprovechamiento de productos forestales, la ganadería extensiva y los proyectos de urbanización (Malizia et al. 2012). Estas modificaciones producen una disminución de la biodiversidad local y afectan las relaciones interespecíficas en las cadenas tróficas al modificar las abundancias de los ítems presas disponibles para los depredadores (Keddy 1992; Barret and Pelles 1999; Love et al. 2000; Massa 2015).

En este trabajo realizamos una caracterización de la ecología trófica de Tyto furcata en seis localidades de Yungas de la Argentina. Sobre la base del análisis del contenido de sus egagrópilas, documentamos la composición y diversidad de los ítems presa depredados por esta rapaz, calculamos la amplitud del nicho trófico y determinamos su aporte en biomasa. El estudio incluyó una caracterización general y un análisis estacional para comparar la dieta durante las temporadas húmeda y seca. Finalmente, se analizaron los parámetros tróficos obtenidos en cada localidad en el contexto de los diferentes grados de transformación de la vegetación original.

\section{Materiales y Métodos}

\section{Área de estudio}

Las egagrópilas fueron colectadas en seis localidades correspondientes a la eco-región de las Yungas (sensu Burkart et al. 1999), una perteneciente a la provincia de Jujuy (Finca Buena Voluntad [FBV], $24^{\circ} 22^{\prime} \mathrm{S}-65^{\circ} 21^{\prime} \mathrm{O}$, 1390 m s.n.m.) y las restantes a la provincia de Tucumán (Residencia UniversitariaParque Biológico [RU], $26^{\circ} 46^{\prime} \mathrm{S}-65^{\circ} 19^{\prime} \mathrm{O}$, 752 m s.n.m.; Ingenio San Pablo [ISP], 265' $\mathrm{S}$ $-65^{\circ} 19^{\prime}$ O, 452 m s.n.m.; Escuela de Agricultura y Sacarotécnia [EAS], $26^{\circ} 46^{\prime} \mathrm{S}-6^{\circ} 19^{\prime} \mathrm{O}, 696$ m s.n.m.; Villa Padre Monti [VPM], 26 ${ }^{\circ} 30^{\prime}$ S - 64 $59^{\circ}$ O, 926 m s.n.m., y Quebrada Los

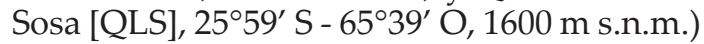
(Figura 1). En este ambiente el clima general es subtropical, con una estación seca que normalmente se extiende de mayo a octubre y un período húmedo que suele extenderse entre noviembre y marzo (Sesma et al. 1998; Brown et al. 2001). Sin embargo, las condiciones locales de temperatura y humedad varían notablemente en función de la latitud, altitud y exposición de las laderas (Burkart et al. 1999). El fuerte gradiente altitudinal en las Yungas permite reconocer cuatro pisos de vegetación diferenciados por su fisonomía y composición florística; tres de ellos están representados en este estudio: a) la Selva Pedemontana, con una altitud que varía entre 400 y $900 \mathrm{~m}$ s.n.m., caracterizada por una temperatura media anual de $21.5^{\circ} \mathrm{C}$, una precipitación anual media de $820 \mathrm{~mm}$, y por el predominio de especies arbóreas como tipa blanca (Tipuana tipu), cebil (Anadenanthera colubrina), pacará (Enterolobium contortisiliquum) y lapacho rosado (Handroanthus impetiginosus); b) la Selva Montana, que se extiende aproximadamente entre 900 y 1600 m s.n.m., y presenta una precipitación anual media de $1800 \mathrm{~mm}$, un clima templado a cálido y húmedo, y una vegetación compuesta mayormente de laurel (Cinnamomum porphyrium, Nectandra pichurim y Ocotea puberula), horco molle (Blepharocalix salicifolius), arrayán (Eugenia uniflora), nogal criollo (Juglans australis) y cedro (Cedrela angustifolia); c) el Bosque Montano, mayormente distribuido entre 1600 y 2500 m s.n.m., con una precipitación anual media de $1100 \mathrm{~mm}$, un clima templado húmedo y representantes arbóreos de la talla del pino del 


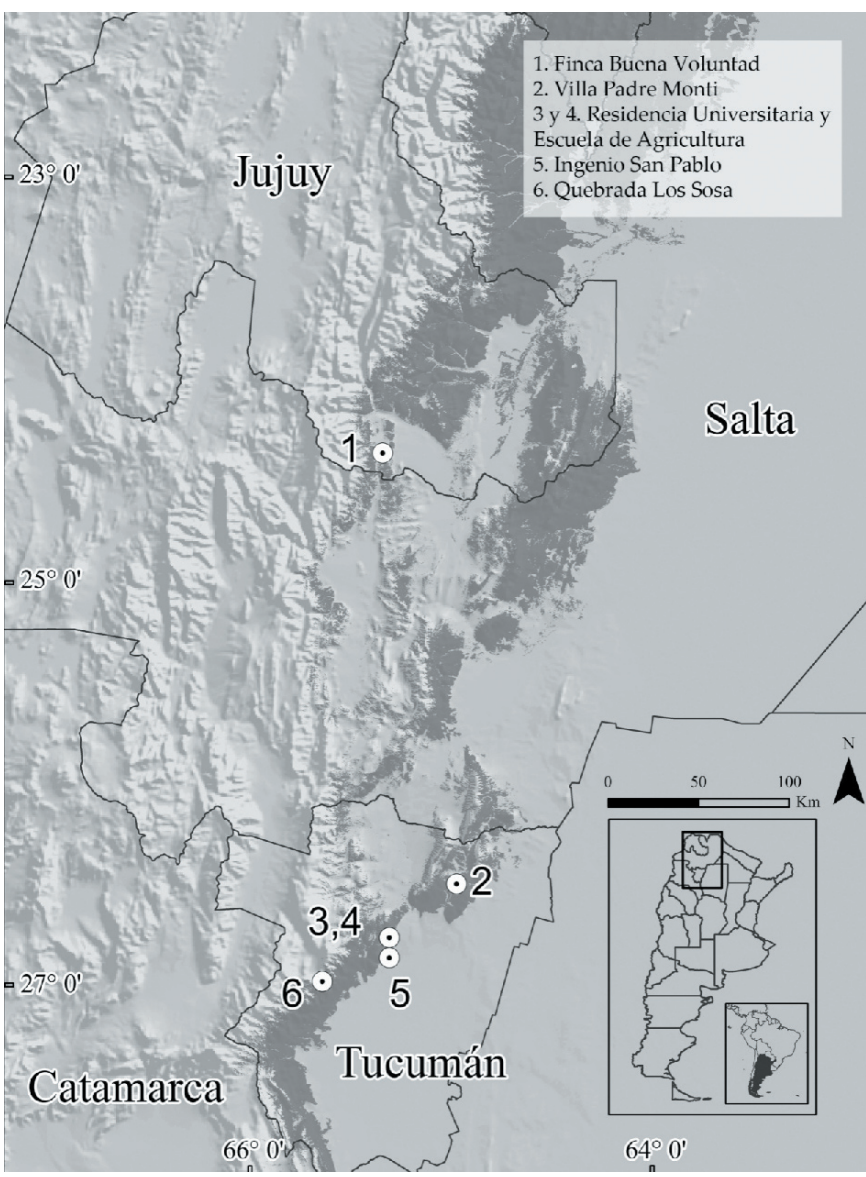

Figura 1. Localidades de colecta de las egagrópilas de Tyto furcata en la ecorregión de las Yungas del noroeste argentino. 1) Finca Buena Voluntad; 2) Villa Padre Monti; 3 y 4) Residencia Universitaria y Escuela de Agricultura; 5) Ingenio San Pablo, y 6) Quebrada Los Sosa.

Figure 1. Collection localities of Tyto furcata pellets in the Yungas ecoregion of northwestern Argentina. cerro (Podocarpus parlatorei), el nogal criollo y el aliso (Alnus acuminata).

Tres de las localidades de colecta corresponden a la Selva Pedemontana (RU, ISP y EAS), dos pertenecen a la Selva Montana (VPM y FBV) y una al Bosque Montano (QLS). Cada una de las localidades presenta diferentes historias de uso del territorio. La localidad RU se encuentra dentro del Parque Sierra de San Javier, una zona protegida que pertenece a la Universidad Nacional de Tucumán, caracterizada por un mosaico de ambientes que incluyen áreas con cultivos de cítricos y un sector ocupado con viviendas, pero dominado por bosques secundarios bien conservados y laderas montañosas con selvas en muy buen estado de conservación. Las demás localidades del pedemonte (ISP y EAS) se encuentran en ambientes mayormente modificados por la transformación de su cobertura natural en áreas de agricultura intensiva, en particular caña de azúcar, desde fines del siglo XIX (Brown and Malizia 2004). Además, en ISP son importantes los ambientes urbanos y periurbanos, mientras que en EAS existe una mayor cobertura de bosques secundarios con distintos grados de conservación. En la Selva Montana, FBV es un área en la que coexisten zonas agrícolo-ganaderas, áreas de extracción forestal y ambientes de bosques secundarios. Mientras tanto, en VPM predomina una matriz de vegetación natural con parches de cultivos y ambientes urbanizados rurales. QLS, la única localidad incluida en el Bosque Montano, se encuentra en un área protegida provincial, un sector dominado por bosques nativos en buen estado de conservación.

\section{Obtención y análisis de datos}

La dieta de Tyto furcata se estudió a partir del análisis de 20 muestras de egagrópilas colectadas entre mayo de 2010 y agosto de 2016 en los sitios de nidificación (RU, EAS, VPM, FBV y QLS) y/o percha (ISP) de las lechuzas. En todos los casos, menos en RU, los nidos/perchas estuvieron situados sobre estructuras construidas por el hombre. En este trabajo se consideraron las muestras que pudieron ser atribuidas sin dudas a Tyto furcata, posiblemente producidas por un ejemplar, una pareja, o una pareja con crías en cada localidad. El número de muestras 
obtenidas para cada localidad no fue uniforme y totalizó seis en RU, una en EAS, dos en ISP, tres en VPM y cuatro en FBV y QLS. Las egagrópilas fueron disectadas en húmedo siguiendo técnicas estándar (Marti et al. 2007), y su contenido se analizó en laboratorio. Los especímenes de pequeños mamíferos recuperados fueron identificados al máximo nivel taxonómico posible a través de su comparación con material de referencia depositado en la Colección de Egagrópilas del Instituto Superior de Correlación Geológica (INSUGEO, Tucumán, Argentina), así como a través de referencias bibliográficas pertinentes. El resto del material recuperado (insectos y aves) fue identificado a nivel de clase.

Para cada muestra se calculó el número mínimo deindividuos (NMI) a partir del conteo de los elementos homólogos pertenecientes a un mismo lado del cráneo o cuerpo (hemimandíbulas, hemimaxilares, élitros, etc.) y el porcentaje aportado a la dieta en términos de biomasa $\left(n_{i} w p_{i} 100 / \Sigma\left[n_{i} w p_{i}\right]\right.$, donde $n_{i}$ es el número de individuos correspondientes a la presa i y wp $\mathrm{p}_{\mathrm{i}}$ es el peso promedio de la presa i) (Marti et al. 2007). Los pesos medios de las presas fueron tomados de la literatura y del catálogo de campo de uno de los autores (JPJ). Además, se calculó la frecuencia relativa (NMI\%) de cada presa dividiendo el número de individuos de cada categoría por el número total de ítems presa registrados, expresado en porcentaje. La amplitud de nicho trófico fue estimada para cada localidad y se caracterizó mediante el Índice de Levins $\left(B=1 / \sum \mathrm{p}_{\mathrm{i}}{ }^{2}\right.$; donde $p_{i}$ es la frecuencia relativa de cada especie), mientras que las comparaciones entre las distintas localidades de muestreo se realizaron mediante el índice de Levins estandarizado ( $\mathrm{Bst}=\mathrm{B}-\mathrm{B}_{\min } / \mathrm{B}_{\max }-\mathrm{B}_{\min }$; donde $\mathrm{B}_{\min }=1$ y $\mathrm{B}_{\max }=$ número total de categorías) (Colwell and Futuyma 1971; Bellocq 2000; Teta et al. 2001). Estos dos últimos índices fueron calculados utilizando la resolución taxonómica de las presas a nivel de especie para pequeños mamíferos no voladores y a nivel de clase para las restantes presas.

El promedio geométrico del peso de las presas (GMPW) se calculó siguiendo a Marti et al. (1993). La diversidad de ítems presa se calculó mediante el índice alfa de Fisher $(\alpha)$ y con el estimador no paramétrico Chao 1. La distribución de abundancias de las presas se cuantificó mediante el índice de ShannonWiener $\left(\mathrm{H}^{\prime}\right)$, que mide equitatividad. Además, en cinco de las seis localidades estudiadas se analizaron las variaciones estacionales en la dieta, y se agruparon las muestras correspondientes a las estaciones húmeda y seca (ver área de estudio). Por otra parte, se calculó la superposición trófica entre estaciones para cada localidad utilizando el índice de Pianka $\left(\mathrm{O}=\sum \mathrm{p}_{\mathrm{ij}} \mathrm{q}_{\mathrm{ij}} / \sqrt{ } \mathrm{p}_{\mathrm{ij}}{ }^{2} \mathrm{q}_{\mathrm{ij}}{ }^{2}\right.$; donde $\mathrm{p}_{\mathrm{ij}} \mathrm{y}_{\mathrm{ij}}$ son las proporciones de la categoría $\mathrm{i}$ en las dos muestras que se comparan), cuyo valor varía entre 0 ( $\sin$ solapamiento) y 1 (solapamiento total).

Para el cálculo de los índices de diversidad se utilizaron los programas PAleontological STatistic (PAST) versión 3.11 (Hammer et al. 2001) y EstimateS versión 9.1.0 (Colwell 2013). Para el análisis de solapamiento de nicho se utilizó la función niche.overlap en el software $\mathrm{R}$ (R Development Core Team 2012), paquete "spaa" (Zhang 2004).

Para las cinco localidades con un NMI mayor a 90 estudiamos la relación entre los parámetros tróficos y el grado de transformación del ambiente mediante regresión lineal simple (previa confirmación de los supuestos que asume este modelo mediante plots de probabilidad normal, prueba de distribución normal [prueba de Shapiro-Wilk] y prueba de homogeneidad de varianzas [prueba de Breusch-Pagan]). Como variable independiente se utilizaron distintas categorías de uso del suelo obtenidas a partir del análisis de imágenes satelitales LandSat (resolución espacial $30 \mathrm{~m}$ ) contemporáneas al año de colecta de las egagrópilas. Las imágenes fueron obtenidas de manera gratuita del Servicio Geológico de Estados Unidos (USGS, earthexplorer.usgs.gov). Todos los procesamientos se realizaron utilizando el sistema de coordenadas geográficas UTM (Universal Transverse Mercator), con base en le modelo elipsoide WGS 84 (World Geodetic System 1984). Las cuatro localidades pertenecientes a Tucumán se encuentran incluidas en el path 231 row 79, mientras que la localidad FBV en Jujuy se encuentra en el path 30 row 78. Para el pre-procesamiento, procesamiento, clasificación y postclasificación de las imágenes se utilizaron los programas ENVI 4.8 y QGIS 2.6 (Quantum GIS Development Team 2014). La interpretación de los diferentes tipos de cobertura se realizó dentro de un buffer circular de $3 \mathrm{~km}$ de radio cuyo centro fue la posición geográfica de cada sitio de muestreo. Esta área de $\sim 28 \mathrm{~km}^{2}$ fue considerada por algunos autores como el área de caza más probable para algunas especies del género Tyto (Andrews 1990). Se llevó a cabo una clasificación supervisada y con la 
Tabla 1. Porcentajes de coberturas del suelo (vegetación natural, cultivo, urbano, agua / río) para las cuatro localidades incluidas en este análisis e índice kappa. [RU]: Residencia Universitaria-Parque Biológico; [ISP]: Ingenio San Pablo; [EAS]: Escuela de Agricultura y Sacarotecnia; [FBV]: Finca Buena Voluntad; [VPM]: Villa Padre Monti; [QLS]: Quebrada Los Sosa.

Table 1. Land cover percentages (natural vegetation, crops, urban, water/rivers) for four localities included in this analysis, with Kappa index.

\begin{tabular}{lccccc}
\hline & Vegetación natural & Cultivo & Agua/Río & Urbano & Kappa \\
\hline RU_2013 & 53.88 & 19.4 & 14.44 & 12.28 & 0.70 \\
ISP_2013 & 8.26 & 78.54 & 0 & 13.2 & 0.80 \\
FBV_2013 & 83.19 & 10.24 & 6.6 & 0 & 0.80 \\
VPM_2013 & 68.31 & 13.27 & 0 & 18.42 & 0.80 \\
QLS_2014 & 88.70 & 0 & 0 & 11.30 & 0.86 \\
\hline
\end{tabular}

ayuda de Google Earth se identificaron las siguientes clases de cobertura: vegetación natural (coberturas boscosas y pastizales), cultivo, urbano y agua (Tabla 1). Para las regresiones, las categorías urbano y agua fueron unificadas bajo el nombre de "no utilizable" dado que es una porción del territorio que no puede ser explotada por los ítems presa. La clasificación supervisada se llevó a cabo con la ayuda del clasificador Support Vector Machine (SVM). Para validar y evaluar la precisión de los mapas de uso del suelo se calculó una matriz de confusión para las cinco clasificaciones y se calculó el índice Kappa. Este índice se puede utilizar como una medida de confiabilidad de la clasificación; varía entre 0 (lo observado en el mapa es debido al azar) y 1 (coincidencia plena entre el mapa y la realidad) (Casagranda 2014). Se obtuvieron cinco matrices, de acuerdo a las cuales, $50 \%$ de las clasificaciones obtuvo un Kappa de 0.7 y el otro $50 \%$ un valor de 0.9 . Los porcentajes de cobertura se obtuvieron con la ayuda de la herramienta "Class Distribution" de ENVI. Los análisis de regresión (ordinary least squares regression) entre las distintas categorías de uso y los parámetros tróficos se realizaron con la ayuda del programa PAleontological STatistic (PAST) versión 3.11 (Hammer et al. 2001).

\section{RESUlTADOS}

A partir del análisis de 713 egagrópilas se registró un NMI total de 2592 ejemplares. El número de ítems presa registrados en la dieta varió entre 7 (EAS) y 14 (FBV); el número de individuos, entre 61 (EAS) y 937 (VPM) (Tabla 2). Los roedores, representados por 18 especies, constituyeron el $98 \%$ del total de presas registradas. En un sentido amplio, incluyendo las seis localidades estudiadas, la dieta de Tyto furcata en Yungas estuvo compuesta principalmente por especies de roedores sigmodontinos de los géneros
Calomys (C. cf. C. fecundus/venustus y C. cf. C. laucha/musculinus), Oligoryzomys (O. brendae y O. cf. O. flavescens) y, en menor medida, Akodon (A. caenosus, A. simulator y A. spegazzinii). Ninguna especie de los restantes géneros de pequeños mamíferos, considerando todas las localidades, superó el $6.5 \%$ de representación en la dieta (Tabla 2). Las aves, presentes en cinco de las seis localidades, constituyeron entre 0.2 y $2.3 \%$ de la dieta, en tanto que los marsupiales didélfidos, los quirópteros y los insectos tuvieron una representación ínfima. Tyto furcata consumió presas comprendidas en un amplio rango de tamaños corporales, desde los $2 \mathrm{~g}$ en insectos hasta los $440 \mathrm{~g}$ en Cavia sp. Losroedoressigmodontinos fueron losquemás aportaron a la dieta en términos de biomasa, y las especies de Calomys, Oligoryzomys y Akodon resultaron los contribuyentes principales en todas las localidades.

En cuatro de las localidades estudiadas (RU, ISP, EAS, VPM), el ítem más consumido por Tyto furcata fue alguna de las especies de Calomys, en todos los casos con una frecuencia superior a $30 \%$. A diferencia de estas localidades, en FBV el elemento dominante en la dieta fue Oligoryzomys brendae (25.3\%) y en QLS la especie más representada fue A. spegazzinii (60.8\%). En dos localidades (RU y EAS), el porcentaje sumado de las dos especies de Calomys superó el 50\%. Los estimadores de diversidad indican que FBV e ISP son los sitios con mayor diversidad en la dieta; allí se registraron valores altos de riqueza y equitatividad. Para QLS también se registraron valores de riqueza elevados, pero la equitatividad en esa localidad fue comparativamente baja. El valor más bajo de diversidad se registró en EAS (Tabla 2). En todas las localidades los pequeños mamíferos aportaron la mayor parte de la biomasa consumida, desde $94 \%$ en ISP hasta $100 \%$ en EAS. En ISPy QLS, las aves aportaron comparativamentemuchabiomasa, igualando, 
Tabla 2. Ítems presa registrados en la dieta de Tyto furcata para seis localidades de la ecorregión de las Yungas del noroeste argentino. [RU]: Residencia Universitaria-Parque Biológico; [ISP]: Ingenio San Pablo; [EAS]: Escuela de Agricultura y Sacarotecnia; [FBV]: Finca Buena Voluntad; [VPM]: Villa Padre Monti; [QLS]: Quebrada Los Sosa. Para cada ítem presa se registra el peso (g), NMI (número mínimo de individuos), \%NMI (porcentaje de participación en la dieta), \%B (porcentaje de biomasa aportado a la dieta), B (amplitud de nicho trófico) y Bst (B estandarizado). Índices de diversidad: $S$ (riqueza), $\alpha$ (índice alpha de Fisher), Chao-1, $\lambda$ (índice de Simpson), $\mathrm{H}^{\prime}$ (índice de Shannon-Wiener), GMPW (promedio geométrico del peso de las presas).

Table 2. Prey items registered in the diet of Tyto furcata in six localities of the Yungas ecoregion, northwest Argentina. For each prey item, we recorded the weight (g), MNI (minimum number of individuals), \%MNI (percent frequency of individuals), \%B (percentual biomass contribution to the diet), B (food niche breadth), Bst (standardized B). Diversity indices: $\mathrm{S}$ (richness), $\alpha$ (Fisher's alpha index), Chao-1, $\lambda$ (Simpson index), H' (Shannon-Wiener index), GMPW (geometric mean prey weight).

\begin{tabular}{|c|c|c|c|c|c|c|c|c|c|c|}
\hline & & & $\mathrm{RU}$ & & & ISP & & & EAS & \\
\hline & Peso & NMI & $\mathrm{NMI} \%$ & $\% B$ & NMI & $\mathrm{NMI} \%$ & $\% B$ & NMI & $\mathrm{NMI} \%$ & $\% \mathrm{~B}$ \\
\hline Mammalia & & 614 & 99.7 & 99.30 & 170 & 97.7 & 94.07 & 61 & 100 & 100 \\
\hline Thylamys sp. & 23.8 & - & - & - & - & - & - & 1 & 1.6 & 1.78 \\
\hline Abrothrix iflutea & 41 & 6 & 1.0 & 2.09 & - & - & - & - & - & - \\
\hline Akodon caenosus & & - & - & - & 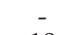 & & & - & & \\
\hline Akodon simulator. & 33.2 & 5 & 0.8 & 1.41 & 10 & 5.7 & 6.15 & 3 & 4.9 & 7.45 \\
\hline Akodon spegazzinii & 19.7 & 45 & 7.3 & 7.54 & 14 & 8.0 & 5.11 & 6 & 9.8 & 8.85 \\
\hline Necromys lactens & 35.7 & - & - & - & - & - & - & - & - & - \\
\hline Necromys sp. & 35.6 & 2 & 0.3 & 0.60 & 8 & 4.6 & 5.28 & - & - & - \\
\hline Oxymycterus paramensis & 56.8 & - & - & - & - & - & - & - & - & - \\
\hline Oxymycterus wayku & 54.1 & - & - & - & - & - & - & - & - & - \\
\hline Andinomys edax & 80 & 2 & 0.3 & 1.36 & - & - & - & - & - & - \\
\hline Holochilus chacarius balnearum & 150 & & - & - & 5 & 2.9 & 13.90 & - & - & - \\
\hline Oligoryzomys brendae & 22.9 & 92 & 14.9 & 17.91 & 16 & 9.2 & 6.79 & 6 & 9.8 & 10.28 \\
\hline Oligoryzomys cf. O. flavescens & 15.6 & 66 & 10.7 & 8.75 & 38 & 21.8 & 10.99 & 10 & 16.4 & 11.68 \\
\hline Calomys cf. C. fecundus/venustus & 30.9 & 113 & 18.3 & 29.69 & 65 & 37.4 & 37.22 & 20 & 32.8 & 46.26 \\
\hline Calomys cf. C. laucha/musculinus & 12.2 & 282 & 45.8 & 29.25 & 6 & 3.4 & 1.36 & 15 & 24.6 & 13.70 \\
\hline Phyllotys anitae & 32.8 & - & - & - & - & - & - & - & - & - \\
\hline Mus sp. & 15.5 & - & - & - & 2 & 1.1 & 0.57 & - & - & - \\
\hline Rattus sp. & 80 & 1 & 0.2 & 0.68 & 4 & 2.3 & 5.93 & - & - & - \\
\hline Cavia sp. & 440 & - & - & - & - & - & - & - & - & - \\
\hline Chiroptera & 20.5 & - & - & - & 2 & 1.1 & 0.76 & - & - & - \\
\hline Aves & 80 & 1 & 0.2 & 0.68 & 4 & 2.3 & 5.93 & - & - & - \\
\hline Insecta & 2 & 1 & 0.2 & 0.02 & & 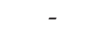 & 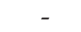 & - & - & 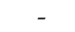 \\
\hline Total & & 616 & & & 174 & & & 61 & & \\
\hline B & & & 3.54 & & & 4.74 & & & 4.61 & \\
\hline Bst & & & 0.21 & & & 0.34 & & & 0.60 & \\
\hline $\mathrm{S}$ & & & 12 & & & 12 & & & 7 & \\
\hline$\alpha$ & & & 2.1 & & & 2.9 & & & 2.04 & \\
\hline Chao-1 & & & 13 & & & 12 & & & 7 & \\
\hline & & & 1.54 & & & 1.92 & & & 1.68 & \\
\hline GMPW & & & 31.01 & & & 28.94 & & & 21.46 & \\
\hline & & & FBV & & & VPM & & & QLS & \\
\hline & & NMI & $\mathrm{NMI} \%$ & $\% \mathrm{~B}$ & NMI & NMI $\%$ & $\% \mathrm{~B}$ & NMI & NMI\% & $\% \mathrm{~B}$ \\
\hline Mammalia & & 702 & 99.3 & 98.92 & 934 & 99.7 & 99.35 & 96 & 98.97 & 96.55 \\
\hline Thylamys sp. & & 23 & 3.3 & 3.55 & 7 & 0.7 & 0.67 & 1 & 1.0 & 0.02 \\
\hline Abrothrix itlutea & & - & - & - & - & - & - & 5 & 5.2 & 8.83 \\
\hline Akodon caenosus & & 148 & 20.9 & 13.34 & - & - & - & 1 & 1.0 & 0.6 \\
\hline Akodon simulator. & & 34 & 4.8 & 7.32 & 141 & 15.0 & 18.72 & 2 & 2.1 & 2.86 \\
\hline Akodon spegazzinii & & - & 100 & 1.02 & 154 & 16.4 & 12.13 & 59 & 60.8 & 50.08 \\
\hline Necromys lactens & & 1 & 0.1 & 0.23 & - & - & - & - & - & - \\
\hline Necromys s & & & - & - & 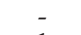 & - & - & - & - & - \\
\hline Oxymycterus paramensis & & 15 & 2.1 & 5.53 & 1 & 0.1 & 0.23 & - & - & - \\
\hline Oxymycterus wayku & & - & - & & - & - & - & 1 & 1.0 & 2.33 \\
\hline Andinomys edax & & 3 & 0.4 & 1.56 & 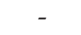 & - & - & 2 & 2.1 & 6.89 \\
\hline Holochilus cha & & - & - & - & - & - & - & - & - & - \\
\hline Oligoryzomys brendae & & 179 & 25.3 & 26.59 & 269 & 28.7 & 24.64 & 9 & 9.3 & 8.90 \\
\hline Oligoryzomys cf. O. flavescens & & 33 & 4.7 & 3.34 & 51 & 5.4 & 3.18 & 9 & 9.3 & 6.05 \\
\hline Calomys cf. C. fecundus/venustus & & 133 & 18.8 & 26.65 & 306 & 32.7 & 37.82 & - & - & - \\
\hline Calomys cf. C. laucha/musculinus & & 127 & 18.0 & 10.05 & 4 & 0.4 & 0.19 & 1 & 1.0 & 0.53 \\
\hline Phyllotys anitae & & & & & - & - & - & 6 & 6.2 & 8.48 \\
\hline Mus sp. & & 1 & 0.1 & 0.10 & - & - & - & - & - & - \\
\hline Rattus sp. & & - & - & - & 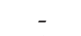 & 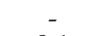 & . & - & - & - \\
\hline Cavo & & 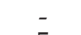 & - & 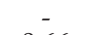 & 1 & 0.1 & 1.76 & - & - & - \\
\hline Chiroptera & & 5 & 0.7 & 0.66 & - & - & - & - & - & - \\
\hline Aves & & 2 & 0.3 & 1.04 & 2 & 0.2 & 0.64 & 1 & 1.0 & 3.45 \\
\hline Insecta & & 3 & 0.4 & 0.04 & $\overline{1}$ & 0.1 & 0.008 & - & 20 & \\
\hline Total & & 707 & & & 937 & & & 97 & & \\
\hline B & & & 5.50 & & & 4.14 & & & 2.53 & \\
\hline Bst & & & 0.35 & & & 0.31 & & & 0.14 & \\
\hline S & & & 14 & & & 11 & & & 12 & \\
\hline & & & 2.5 & & & 1.75 & & & 3.60 & \\
\hline Chao-1 & & & 14.5 & & & 12.5 & & & 15.3 & \\
\hline & & & 1.9 & & & 1.56 & & & 1.45 & \\
\hline GMPW & & & 25.52 & & & 33.44 & & & 26.93 & \\
\hline
\end{tabular}


e incluso superando, a varias de las especies de roedores (Tabla 2). El aporte de Calomys cf. C. fecundus/venustus superó siempre el $26 \%$, y fue la especie que más contribuyó en cuanto a biomasa consumida por $T$. furcata. En la única localidad donde este roedor estuvo ausente (QLS), Akodon spegazzinii fue la especie con mayor aporte $(60.8 \%)$. Como una consecuencia de su alta representación, tanto en RU como en EAS las dos especies de Calomys en conjunto contribuyeron en casi $60 \%$ de la biomasa total consumida. VPM y FBV, las dos localidades de la Selva Montana, muestran diferencias importantes en cuanto a la biomasa aportada por C. cf. C. laucha/musculinus, siendo notablemente mayor en FBV. Los valores de amplitud de nicho trófico variaron entre 2.53 (QLS) y 5.50 (FBV), en tanto que este ítem estandarizado lo hizo entre 0.14 (QLS) y 0.60 (EAS) (Tabla 2). El promedio geométrico de los pesos de las presas consumidas por Tyto furcata en Yungas de Argentina estuvo comprendido entre $21.46 \mathrm{~g}$ (EAS) y $33.44 \mathrm{~g}$ (VPM).

Se observaron cambios estacionales en la composición de especies para cada localidad. Estos cambios involucraron entre 3 y 6 ítems registrados sólo en una de las dos estaciones consideradas. En términos generales. los ítems más comúnmente consumidos por $T$. furcata (e.g., las especies de Akodon, Calomys y Oligoryzomys) estuvieron presentes en ambas estaciones del año (Apéndice 1 en Información Suplementaria). En tres de las localidades (RU, ISP, FBV), los estimadores de riqueza y equitatividad fueron mayores para la estación húmeda (Tabla 3). Estos valores más elevados se explican, al menos en parte, por el aporte de ítems presa comparativamente infrecuentes en la dieta de esta rapaz, como quirópteros, aves e insectos (en RU y FBV), pero también por la presencia de especies de roedores consumidos sólo en esta estación del año. Las localidades con mayor riqueza y equitatividad en la estación seca fueron VPM y QLS, principalmente por el mayor aporte de roedores (Tabla 3). En estas localidades, el estimador Chao-1 fue muy superior en la estación seca con respecto a la estación húmeda. La abundancia de los pequeños mamíferos terrestres en la dieta de Ty to furcata fue notable tanto en la estación húmeda como en la seca, con valores comparativamente altos en todas las localidades estudiadas (Apéndice 1 en Información suplementaria). Sin embargo, la abundancia de algunas especies varió de manera sensible entre estaciones dentro de una misma localidad, como, por ejemplo, Calomys cf. C. laucha/musculinus y Oligoryzomys brendae en RU, de Necromys sp. en ISP, de Akodon simulator y Oxymycterus paramensis en FBV, de $A$. simulator en VPM, y de O. brendae y Phyllotis anitae en QLS. En todos estos casos, la representación fue el doble, o más, en una estación respecto de otra (Apéndice 1 en Información Suplementaria).

La amplitud de nicho trófico fue mayor durante la estación húmeda en cuatro de las cinco localidades analizadas (RU, ISP, FBV, QLS), y alcanzó en RU la mayor diferencia entre estaciones. Sin embargo, la versión estandarizada de este índice confirmó este patrón sólo en el caso de RU y QLS, al mismo tiempo que agrega a VPM (Tabla 3). El solapamiento trófico entre estaciones alcanzó valores de 0.77 en RU, 0.96 en ISP y 0.98 en FBV, VPM y QLS.

Los resultados obtenidos no indican una relación clara entre el grado de modificación antrópica y los parámetros tróficos analizados para las cinco localidades estudiadas. Las regresiones no fueron estadísticamente significativas para las comparaciones de

Tabla 3. Parámetros tróficos en la dieta de Tyto furcata para cada localidad durante las estaciones húmeda (EH) y seca (ES). [RU]: Residencia Universitaria-Parque Biológico; [ISP]: Ingenio San Pablo; [EAS]: Escuela de Agricultura y Sacarotecnia; [FBV]: Finca Buena Voluntad; [VPM]: Villa Padre Monti; [QLS]: Quebrada Los Sosa.

Table 3. Trophic parameters in the diet of Tyto furcata for each locality during wet and dry seasons. Abbreviations are the same as in Table 1.

\begin{tabular}{lcccccccccc}
\hline \multicolumn{1}{c}{ Parámetros } & \multicolumn{2}{c}{ RU } & \multicolumn{2}{c}{ ISP } & \multicolumn{2}{c}{ FBV } & \multicolumn{2}{c}{ VPM } & \multicolumn{2}{c}{ QLS } \\
\multicolumn{1}{c}{ tróficos } & EH & ES & EH & ES & EH & ES & EH & ES & EH & ES \\
\hline NMI & 223 & 393 & 133 & 41 & 455 & 252 & 208 & 729 & 26 & 71 \\
B & 4.45 & 2.71 & 4.74 & 4.48 & 5.94 & 4.7 & 3.81 & 4.19 & 2.64 & 2.46 \\
Bst & 0.43 & 0.24 & 0.34 & 0.44 & 0.38 & 0.46 & 0.47 & 0.32 & 0.33 & 0.15 \\
S & 9 & 8 & 12 & 9 & 14 & 9 & 7 & 11 & 6 & 11 \\
$\alpha$ & 1.88 & 1.42 & 3.2 & 3.56 & 2.73 & 1.82 & 1.4 & 1.84 & 2.45 & 3.64 \\
Chao-1 & 10.5 & 8 & 12 & 12 & 17 & 9 & 7 & 17 & 6.5 & 13 \\
$\lambda$ & 0.77 & 0.63 & 0.79 & 0.78 & 0.83 & 0.79 & 0.74 & 0.76 & 0.62 & 0.6 \\
H' & 1.62 & 1.35 & 1.91 & 1.79 & 1.97 & 1.68 & 1.5 & 1.56 & 1.3 & 1.45 \\
GMPW & 28.93 & 24.47 & 28.95 & 30.71 & 25.52 & 23.2 & 23.58 & 33.41 & 22.01 & 29.15 \\
\hline
\end{tabular}


los parámetros tróficos en función de la proporción de ambientes naturales $(P=0.500$ para $B, P=0.395$ para Bst, $P=0.814$ para $S$, $P=0.843$ para $\alpha, P=0.092$ para Chao- $1, P=0.291$ para $\mathrm{H}^{\prime}$ y $P=0.723$ para $\mathrm{GMPW}$ ) ni de la proporción de los cultivos $(P=0.334$ para $\mathrm{B}$, $P=0.271$ para Bst, $P=0.924$ para $S, P=0.996$ para $\alpha, P=0.196$ para Chao- $1, P=0.127$ para $\mathrm{H}^{\prime} \mathrm{y}$ $P=0.996$ GMPW).

\section{DiscUSIÓN}

Por su abundancia y amplia distribución en el continente americano, la ecología trófica de Tyto furcata fue caracterizada en detalle (e.g., Anderson and Long 1961; Cerpa and Yáñez 1981; De Santis et al. 1994; David 1996; Jordão et al. 1996). Sin embargo, aún existen ambientes naturales para los que la información sobre este tópico es escasa o nula. En la Argentina, la mayor parte de estas investigaciones se realizaron en la Región Pampeana y, en menor medida, en las regiones Mesopotámica y Patagónica (Massoia 1989, 1990; Pardiñas and Cirignoli 2002; Trejo et al. 2005; Leveau et al. 2006; Bó et al. 2007; Teta et al. 2010; González Fischer et al. 2012; Massa 2015), con tan sólo unos pocos aportes para el noroeste argentino (e.g., Gómez et al. 2012; Nanni et al. 2012). Asimismo, casi la totalidad de estos estudios se enfocaron en ambientes abiertos, y existen pocos trabajos centrados en caracterizar la dieta de esta rapaz en ecosistemas boscosos.

Las especies de lechuzas de este género son rapaces, con una dieta generalista especializada en el consumo de pequeños mamíferos y con una conducta de caza oportunista. Así, el análisis del contenido de sus egagrópilas es considerado una buena aproximación a la disponibilidad de presas que ofrecen las comunidades de pequeños mamíferos de una localidad en un momento determinado (Massa 2015; Andrade et al. 2015). Bernard et al. (2010) demostraron que la frecuencia de una determinada especie en la dieta de Tyto alba depende de su abundancia real en la comunidad, por lo que una caracterización adecuada de la ecología trófica de esta lechuza debe abordarse tanto desde una perspectiva espacial como temporal. Este fue el énfasis de nuestro estudio, que incluyó seis localidades pertenecientes a los tres pisos altitudinales de las Yungas y con muestras representativas de estaciones diferentes obtenidas a lo largo de varios años. Sin embargo, el alcance de nuestros resultados debe ser interpretado en el contexto del número limitado de individuos estudiados. Nuestros resultados en los bosques húmedos de Yungas coinciden en términos generales con aquellos reportados para otras áreas de América del Sur (e.g., Pillado and Trejo 2000; Bonvicino and Bezerra 2003; Carmona and Rivadeneira 2006; Leveau et al. 2006; Bó et al. 2007; Donadío et al. 2009; González Fischer et al. 2012; Gómez et al. 2012; Nanni et al. 2012), con un espectro relativamente amplio de ítems presa que incluye mamíferos, aves y artrópodos, y una selección fuerte por roedores cricétidos. Asimismo, este grupo fue el que más aportó a la dieta en términos de biomasa, tanto en el análisis general como en el estacional.

La dominancia en la dieta de Tyto furcata de una o de dos especies de Calomys en cinco de las seis muestras estudiadas fue reconocida también en otro ambiente boscoso del noroeste argentino, el Chaco Seco (Gómez et al. 2012; Nanni et al. 2012). Sin embargo, y a diferencia de las muestras chaqueñas, en el caso de las Yungas, esta dominancia es compartida de forma parcial con dos especies de Oligoryzomys (Tabla 2). Al mismo tiempo, resulta notable en nuestro estudio la ínfima participación de roedores caviomorfos en la dieta. Si bien su consumo por parte de esta rapaz es siempre muy inferior a los roedores cricétidos (e.g., Trejo and Ojeda 2004; Leveau et al. 2006; Donadío et al. 2009; Teta et al. 2010; Gómez et al. 2012; Nanni et al. 2012; ver Aliaga-Rossel and Tarifa 2005), en las Yungas, esta situación fue extrema, con tan sólo un individuo de Cavia sp. entre casi 2600 pequeños mamíferos consumidos.

El promedio geométrico de los pesos de las presas estuvo comprendido dentro del amplio rango de variación registrado para las presas consumidas por esta lechuza en América del Sur y por debajo del valor medio de $45 \mathrm{~g}$ propuesto por Marti et al. (1993) para la Región Neotropical. Esta variabilidad evidencia el oportunismo de este depredador y la dependencia que tiene su dieta de la disponibilidad local de las presas (ampliamente conocida a partir de relevamientos realizados por los autores y de la bibliografía disponible). Sin embargo, el tamaño de las presas consumidas depende también de otros factores, tales como la vulnerabilidad de las presas y la estrategia de caza de esta lechuza (Bellocq 1998; Bo et al. 2007). Esto podría explicar, al menos en parte, la frecuencia elevada que se registró en las especies de Calomys. Como otras especies 
del género, Tyto furcata es un depredador que selecciona ambientes abiertos, y es en estos hábitats donde las especies de Calomys son más abundantes y donde, probablemente, sean más vulnerables. La amplitud de nicho trófico registrada en Yungas estuvo dentro de los valores generales para el continente sudamericano, con un promedio de B de 4.28 (Bst=0.48) para latitudes templadas, y de 4.61 (Bst=0.38) para latitudes tropicales (Marti et al. 1993). Bellocq (2000) reportó para Argentina un rango entre 1.37 y 7.49 (Bst: entre 0.05 y 0.69 ) y valores promedio de 4.07 $(B s t=0.25)$ para latitudes subtropicales y de 4.03 (Bst=0.35) en áreas templadas, aunque en este último caso, los índices fueron calculados a nivel de orden. Los valores de amplitud de nicho trófico (y su versión estandarizada) en Yungas fueron más elevados si se los compara con localidades del Chaco Seco (Gómez et al. 2012), con todas las localidades con un B superior a 2.50 y un Bst por encima de 0.21 (B: entre 1.77 y 6.29; Bst: entre 0.11 y 0.66 en el Chaco Seco). Entre los datos disponibles para otras localidades situadas en latitudes subtropicales de Argentina, en ambientes de Chaco Húmedo y de los Esteros del Iberá también se indicaron valores inferiores a los reportados en este trabajo (B: entre 1.49 y 4.91; Bst: entre 0.10 y 0.45) (Pardiñas et al. 2005). En algunas de las localidades de Yungas estudiadas en este trabajo se observa una tendencia general a la disminución de la amplitud de nicho trófico durante la estación seca, lo que muy probablemente refleja las variaciones estacionales en la abundancia de pequeños mamíferos terrestres que muestran tamaños poblacionales más altos durante la época invernal. Durante el verano, la baja disponibilidad de estos mamíferos llevaría a esta lechuza a adoptar una dieta más generalista, con mayor consumo de quirópteros, aves, e insectos (Nanni et al. 2012).

Del mismo modo que con la amplitud de nicho trófico, la tendencia hacia una mayor diversidad en la dieta durante la estación húmeda en algunas de las localidades puede vincularse al mayor consumo de aves e insectos que en la estación seca. Nanni et al. (2012) reportaron tendencias similares, aunque mucho más marcadas, para una localidad de Chaco Seco en la provincia de Santiago del Estero. Un comportamiento similar se ha detectado en estudios realizados en diversas localidades de la región pampeana (González Fischer et al. 2012) así como en el sudeste de Brasil (Motta-Junior and Alho 2000). En contraste con lo observado en localidades del Chaco Seco estudiadas por Gómez et al. (2012), quienes reportaron pérdida de diversidad en correlación con la transformación de la vegetación original en agroecosistemas, en las localidades de Yungas, la proporción de ambientes naturales y/o de cultivos (al menos dentro de un buffer de $3 \mathrm{~km}$ ) no parece tener una incidencia importante sobre ninguno de los parámetros tróficos. Los hábitos de caza de esta especie constituyen un factor adicional de sesgo en nuestro análisis. Dado que Ty to furcata selecciona ambientes abiertos, la presencia y abundancia de las especies características de bosques podrían estar subestimadas en la muestra, más allá del estado de conservación del ambiente. Sin embargo, es evidente que el marco ambiental general de cada localidad tiene una influencia marcada sobre los ítems presas que consume esta rapaz.

Aunque nuestros resultados constituyen un punto de partida importante para el estudio de las características del nicho trófico de Tyto furcata en los bosques de Yungas, resulta evidente la necesidad de estudios adicionales para dilucidar numerosos aspectos relacionados con la ecología de este depredador en este ambiente. Así, una comprensión integral de la ecología trófica de esta rapaz y su vinculación con la actual transformación del paisaje precisa de estudios independientes sobre la abundancia relativa de sus presas, así como una cuantificación detallada de la alteración de la matriz ambiental a distintas escalas.

Agradecimientos. Agradecemos a todas aquellas personas que colaboraron en la obtención de las muestras en el campo. C. Jayat realizó la mayor parte de las colectas de egagrópilas en la Finca Buena Voluntad, Jujuy. S. Nanni y D. García López colaboraron en las tareas de campo en Villa Padre Monti, Tucumán. P. Blendinger gentilmente cedió una muestra de egagrópilas obtenida en Quebrada Los Sosa, Tucumán. Este trabajo se llevó a cabo con el apoyo institucional del Instituto de Ecología Regional (IER-UNT- CONICET), la Cátedra de Paleontología de la Facultad de Ciencias Naturales e Instituto Miguel Lillo, el Instituto Superior de Correlación Geológica (INSUGEO; UNT- CONICET) y el Instituto de Ambiente de Montaña y Regiones Áridas (IAMRA- UNdeC). Parte de las tareas de campo fueron solventadas con fondos obtenidos en el marco del proyecto PICT 2012-0050 del Foncyt. 


\section{REFERENCIAS}

Aliaga Rossel, E., and T. Tarifa. 2005. Cavia sp. como principal presa de la lechuza de campanario (Tyto alba) al final de la estación seca en una zona intervenida al norte del Departamento de La Paz, Bolivia. Ecología en Bolivia 40:35-42.

Álvarez Castañeda, S. T., N. Cárdenas, and L. Méndez. 2004. Analysis of mammal remains from owl pellets (Tyto alba), in a suburban area in Baja California. Journal of Arid Environments 59:59-69.

Anderson, S., and C.A. Long. 1961. Small mammals in pellets of barn owls from Miñaca, Chihuahua. American Museum Novitiates 2052:1-3.

Andrade, A., J. F. Saraiva de Menezes, and A. Monjeau. 2016. Are owl pellets good estimators of prey abundance? Journal of King Saud University- Science 28:239-244.

Andrews, P. 1990. Owls, caves, and fossils: predation, preservation, and accumulation of small mammal bones in caves, with an analysis of the Pleistocene cave faunas from Westburysub-Mendip, Somerset, UK. University of Chicago Press (Eds.), Chicago.

Barret, G. W., and J. D. Peles. 1999. Landscape ecology of small mammals. Springer (Eds.), New York, Estados Unidos.

Bellocq, M. I. 1998. Prey selection by breeding and nonbreeding barn owls in Argentina. The Auk 115:224-229.

Bellocq, M. I. 2000. A review of the trophic ecology of the Barn Owl in Argentina. Journal Raptor Research 34:108119.

Bernard, N., D. Michelat, F. Raoul, J. P. Quéré, P. Delattre, and P. Giraudoux. 2010. Dietary response of Barn Owls (Tyto alba) to large variations in populations of common voles (Microtus arvalis) and European water voles (Arvicola terrestris). Canadian Journal of Zoology 88:416-426.

Bó, M. S., A. V. Baladrón, and L. M. Biondi. 2007. Ecología trófica de Falconiformes y Strigiformes: Tiempo de Síntesis. Hornero 22:97-115.

Bonvicino, C. R., and A. M. R. Bezerra. 2003. Use of regurgitated pellets of Barn Owl (Tyto alba) for inventorying small mammals in the Cerrado of Central Brazil. Studies on Neotropical Fauna and Environment 38:1-5.

Brown, A. D., H. R. Grau, L. R. Malizia, and A. Grau. 2001. Argentina. Pp. 623- 659 en M. Kappelle and A. D. Brown (eds.). Bosques nublados del geotrópico. Instituto Nacional de Biodiversidad, San José, Costa Rica.

Brown, A. D., and L. R. Malizia. 2004. Las selvas pedemontanas de las Yungas: en el umbral de la extinción. Revista Ciencia Hoy 14:53-63.

Burkart, R., N. Bárbaro, R. Sánchez, and D. Gómez. 1999. Eco-regiones de la Argentina. Administración de Parques Nacionales, Programa de Desarrollo Institucional Ambiental, Buenos Aires, Argentina.

Casagranda, M. E. 2014. Análisis retrospectivo: Relación entre cambios de uso de la tierra y cobertura y brote de leishmaniasis cutánea en Vinalito, provincia de Jujuy. Magister en Aplicaciones Espaciales de Alerta y Respuesta Temprana a Emergencias. Universidad Nacional de Córdoba, Córdoba. Argentina. Pp. 75.

Carmona, E., and M. Rivadeneira. 2006. Foods habits of the barn owl Tyto alba in National Reserve Pampa del Tamarugal, Atacama Desert, North Chile. Journal of Natural History 40:473-483.

Cerpa, C., and J. L. Yáñez. 1981. Variación estacional de la dieta de Tyto alba (Gray, 1929) en la zona mediterránea de Chile Central. Boletín del Museo Nacional de Historia Natural de Chile Central 38:137-146.

Colwell, R. K. 2013. EstimateS: Statistical estimation of species richness and shared species from samples. Version 9. URL: purl.oclc.org/estimates.

Colwell, R. K., and D. J. Futuyma. 1971. On the measurement of niche breadth and overlap. Ecology 52:567-576.

David, P. G. 1996. Nestings and food habitats of barn owls in south Florida. Florida Field Naturalist 24:93-136.

De Santis, L. J. M., N. G. Basso, J. L. Noriega, and M. F. Grossman. 1994. Explotación del recurso trófico por la lechuza de los campanarios (Tyto alba) en el oeste de Chubut, Argentina. Studies on neotropical fauna and environment 29: 43-47.

Donadío, E., M. L. Merino, and M. J. Bolgeri. 2009. Diets of two coexisting owls in the High Andes of Northwestern Argentina. Ornitología Neotropical 20:137-141.

Gómez, M. D., G. Fontanarrosa, P. E. Ortiz, and J. P. Jayat. 2012. Pequeños mamíferos predados por la Lechuza de Campanario (Tyto alba) en la ecorregión del Chaco Seco en el noroeste argentino. Hornero 27:127-135.

González Fischer, G., C. M. Baldi, M. Codesido, and D. Bilenca. 2012. Seasonal variations in small mammal- landscape associations in temperate agroecosystems: a study case in Buenos Aires province, central Argentina. Mammalia 76: 399-406.

Hammer, O., D. A. T. Harper, and P. D. Ryan. 2001. PAST: Paleontological Statistics Software Package for Education and Data Analysis. Paleontología Electrónica 4:1-9.

Jaksic, F. M., J. L Yánez, and E. R. Fuentes. 1981. Assessing a small mammal community in Central Chile. Journal of Mammalogy 62:391-396.

Jaksic, F. M., and C. D. Marti. 1981. Trophic ecology of Athene owl in mediterranean- type ecosystems: a comparative analysis. Canadian Journal of Zoology 59:2331-2340.

Jordão, F. S., M. L. Reis, and D. S. Louazada. 1996. Análise do conteúdo de pelotas de Tyto alba (Strigiformes, Tytonidae) na estação Ecológica de Águas Emendadas (Brasília, DF). Pp. 207-209 in L. L. Leite and C. H. Saito (eds.). Contribuição ao Conhecimento Ecológico do Cerrado, Brasília. Universidade de Brasília, Brasil.

Keddy, P. A. 1992. Assembly and response rules: two goles for predictive community ecology. Journal of Vegetation Science 3:157-164. 
Leveau, L. M., P. Teta, R. Bogdaschewsky, and U. F. J. Pardiñas. 2006. Feedings habits of the Barn Owl (Tyto alba) along a longitudinal-latitudinal gradient in Central Argentina. Ornitología Neotropical 17:353-362.

Love, R. A., C. Webon, D. E. Glue, and S. Harris. 2000. Changes in the food of British Barn Owls (Tyto alba) between 1974 and 1997. Mammal Review 30:107-129.

Malizia, L., S. Pacheco, C. Blundo, and A. D. Brown. 2012. Caracterización altitudinal, uso y conservación de las Yungas Subtropicales de Argentina. Ecosistemas 21:53-73.

Marti, C. D., E. Korpimaki, and F. M. Jaksic. 1993. Trophic structure of raptor communities: a three- continent comparison and synthesis. Current Ornithology 10:47-137.

Marti, C. D., M. Bechard, and F. M. Jaksic. 2007. Food habits. Pp. 129-152 in D. M. Bird and K. L. Bildstein (eds.). Raptor research and management techniques. Hancock House, Blaine, Estados Unidos.

Massa, C. 2015. Ecología del Paisaje: comunidades de pequeños roedores de la Provincia de Entre Ríos. Tesis Doctoral. Universidad de Buenos Aires, Buenos Aires. Pp. 133.

Massoia, E. 1987. Algunos mamíferos recolectados en Guampacha, Guasayán, Santiago del Estero. Boletín Científico, Asociación para la Protección de la Naturaleza 1:1-12.

Massoia, E. 1988. Análisis de regurgitados de Tyto alba de Humaitá, Departamento Cerrillos, Provincia de Salta. Boletín Científico, Asociación para la Protección de la Naturaleza 5:6-11.

Massoia, E. 1989. Animales depredados por Tyto alba tuidara en la ciudad de San Miguel, partido de General Sarmiento, provincia de Buenos Aires. Boletín Científico, Asociación para la protección de la Naturaleza 15:2-7.

Massoia, E. 1990. Roedores depredados por Tyto alba tuidara en Mar del Tuyú, partido de General Lavalle, provincia de Buenos Aires. Boletín Científico, Asociación para la Protección de la Naturaleza 17:17-20.

Massoia, E., S. Heinonen Fortabat, and A. J. Diéguez. 1997a. Análisis de componentes mastozoológicos y ornitológicos en regurgitados de Tyto alba de Estancia Guaycolec, Departamento Pilcomayo, provincia de Formosa, República Argentina. Boletín Científico, Asociación para la Protección de la Naturaleza 32:12-16.

Massoia, E., S. Tiranti, and A. J. Diéguez. 1997b. Pequeños mamíferos depredados por Tyto alba en la provincia de La Pampa según sucesivas recolecciones. Boletín Científico, Asociación para la Protección de la Naturaleza 32:19-21.

Massoia, E., H. Pastore, and J. C. Chebez. 1999. Mamíferos depredados por Tyto alba en los departamentos de Gral. Ocampo y Rosario V. Peñaloza, Provincia de La Rioja. Boletín Científico, Asociación para la Protección de la Naturaleza 37:17-20.

Motta-Junior, J. C., and C. J. R. Alho. 2000. Ecologia alimentar de Athene cunicularia e Tyto alba (Aves: Strigiformes) nas Estações Ecológica de Jataí e Experimental de Luiz Antônio. Pp. 303-316 in J. E. Santos and J. S. R. Pires (eds.). Estação Ecológica de Jataí. Volume I. RIMA Editora, São Carlos, Brasil.

Nanni, A. S., P. E. Ortiz, J. P. Jayat, and E. Martín. 2012. Variación estacional de la dieta de la Lechuza de Campanario (Tyto alba) en un ambiente perturbado del Chaco Seco argentino. Hornero 27:149-157.

Oviedo de la Vega, A. R. 1962. Breve estudio sobre la Tyto alba tuidara (Gray). Cuadernos de la Revista de la Facultad de Ciencias Naturales de la Universidad Nacional de Tucumán 3:7-27.

Pardiñas, U. F. J., and S. Cirignoli. 2002. Bibliografía comentada sobre los análisis de egagrópilas de aves rapaces en Argentina. Ornitología Neotropical 13:31-59.

Pardiñas, U. F. J., P. Teta, and S. Heinonen Fortabat. 2005. Vertebrate prey of the Barn Owl (Tyto alba) in subtropical wetlands of northeastern Argentina and eastern Paraguay. Journal of Raptor Research 39:65-69.

Pillado, M. S., and A. Trejo. 2000. Diet of the barn owl (Tyto alba tuidara) in northwestern Argentine Patagonia. Journal of Raptor Research 34:334-338.

R Development Core Team. 2011. R: A language and environment for statistical computing. Vienna, Austria: The R Foundation for Statistical Computing.

Sesma, J. S., E. Y. Guido, and M. E. Puchulu. 1998. Clima de la provincia de Tucumán. Pp. 41-46 en M. Gianfrancisco, M. Puchulu, J. Durango de Cabrera and G. Aceñolaza (eds.). Geología de Tucumán. San Miguel de Tucumán: Colegio de Graduados en Ciencias Geológicas de Tucumán, Tucumán, Argentina.

Soncini, R., H. Salas, and L. Marcus. 1985. Alimentación de la lechuza de los campanarios (Tyto alba) en San Miguel de Tucumán. Historia Natural 5:49-54.

Teta, P., C. Panti, A. Andrade, and A. Pérez. 2001. Amplitud y composición de la dieta de Bubo virginianus (Aves: Strigiformes: Strigidae) en la Patagonia noroccidental Argentina. Boletín de la Sociedad Biológica de Concepción 72:131-138.

Teta, P., C. M. González-Fischer, M. Codesido, and D. Bilenca. 2010. A contribution from Barn Owls pellets analysis to know micromammalian distributions in Buenos Aires province, Argentina. Mammalia 74:97-103.

Trejo, A., and V. Ojeda. 2004. Diet of Barn Owls (Tyto alba) in forested habitats of northwestern Argentine Patagonia. Ornitología Neotropical 15:307-311.

Trejo, A., M. Kun, M. Sahores, and S. Seijas. 2005. Diet overlap and prey size of two owls in the forest -steppe ecotone of Southern Argentina. Ornitología Neotropical 16:539-546.

Zhang, J. T. 2004. Quantitative Ecology. Science Press, Beijing (in Chinese), China. 